\title{
Fixed point theorems for decreasing operators in ordered Banach spaces with lattice structure and their applications
}

\author{
Xingchang Li ${ }^{*}$ and Zhihao Wang
}

"Correspondence:

Ixctsq@mail.163.com

Center for Economic Research,

Harbin University of Commerce,

Harbin, 150028, China

\begin{abstract}
This paper presents some theorems of the fixed point for decreasing operators in Banach spaces with lattice structure. The results are applied to nonlinear second-order elliptic equations.

MSC: $47 \mathrm{H} 10 ; 34 \mathrm{~B} 15$
\end{abstract}

Keywords: decreasing operators; lattice structure; nonlinear; elliptic equations

\section{Introduction and preliminaries}

The fixed point theory for monotone operators in ordered Banach spaces has been investigated extensively in the past 30 years [1-8]. Many new fixed point theorems have been proved under the nonlinear contractive condition by using the theorem of cone and monotone iterative technique. These results have been applied to study the ordinary differential equations, partial differential equations, and integral equations.

In this paper, we investigate decreasing operators in ordered Banach spaces with lattice structure. The theoretical results of fixed points are extended by using the famous Schauder fixed point theorem for the operators. We weaken the conditions of the Schauder fixed point theorem. The results of this paper have no need for the closed bounded and convex property of domains for the operators. To demonstrate the applicability of our results, we apply them to study a problem of nonlinear second-order elliptic equations in the final section of the paper, and the existence of solution is obtained.

Let $E$ be a Banach space and $P$ be a cone of $E$. We define a partial ordering $\leq$ with respect to $P$ by $x \leq y$ if only if $y-x \in P$. A cone $P \subset E$ is called normal if there is a constant $N>0$ such that $\theta \leq x \leq y$ implies $\|x\| \leq N\|y\|$ for all $x, y \in E$. The least positive constant $N$ satisfying the above inequality is called the normal constant of $P$.

Let $E$ be a partially ordered set. We call $E$ a lattice in the partial ordering $\leq$. For arbitrary $x, y \in E, \sup \{x, y\}$ and $\inf \{x, y\}$ exist. One can see [7] for the definition and the properties of the lattice.

Let $D \subset E$, the operator $A: D \longrightarrow E$ is said to be an increasing operator if $x, y \in D, x \leq y$, implies $A x \leq A y$; the operator $A: D \longrightarrow E$ is said to be a decreasing operator if $x, y \in D$, $x \leq y$, implies $A y \leq A x$.

Lemma 1.1 [9] Let E be a real Banach space, $D \subset E$ be nonempty, closed bounded convex, and $A: D \rightarrow D$ be condensing. Then $A$ has a fixed point in $D$.

๑) $2013 \mathrm{Li}$ and Wang; licensee Springer. This is an Open Access article distributed under the terms of the Creative Commons Attribution License (http://creativecommons.org/licenses/by/2.0), which permits unrestricted use, distribution, and reproduction in any medium, provided the original work is properly cited. 
Lemma 1.2 [10] Let E be a real Banach space, $D \subset E$ be nonempty, closed bounded convex, and $A: D \rightarrow D$ be completely continuous. Then $A$ has a fixed point in $D$.

Lemma 1.3 [11] Let E be a real Banach space, $D \subset$ E be nonempty, closed bounded convex, and $A: D \rightarrow D$ be strict-set-contraction mappings. Then $A$ has a fixed point in $D$.

Remark 1 Lemma 1.1 is the famous Sadovskii fixed point theorem; Lemma 1.2 is the famous Schauder fixed point theorem; Lemma 1.3 is the famous Darbo fixed point theorem.

\section{Main results}

Theorem 2.1 Let E be an ordered Banach space with lattice structure, $D \subset E$ be bounded, and $A: D \longrightarrow D$ be a decreasing and condensing operator. Then the operator $A$ has a fixed point in $D$.

Proof For any $x \in D$, since $A: D \longrightarrow D$, we have $A x \in D$.

Since $E$ is a Banach space with lattice structure and $D \subset E$ is bounded, there exists $u_{0} \in D$ such that

$$
\inf \{A x, x\}=u_{0} .
$$

That is,

$$
u_{0} \leq A x, \quad u_{0} \leq x .
$$

Since $A$ is a decreasing operator, we have

$$
A^{2} x \leq A u_{0}, \quad A x \leq A u_{0} .
$$

(2.1) and (2.2) show that

$$
u_{0} \leq A u_{0}
$$

Similar to the proof of (2.3), there exists $v_{0} \in D$ such that

$$
\sup \{A x, x\}=v_{0} .
$$

That is,

$$
A x \leq v_{0}, \quad x \leq v_{0} .
$$

Since $A$ is a decreasing operator, we have

$$
A v_{0} \leq A^{2} x, \quad A v_{0} \leq A x .
$$

(2.4) and (2.5) show that

$$
A v_{0} \leq v_{0} .
$$


(2.3) and (2.6) together with $u_{0} \leq v_{0}$ show that

$$
u_{0} \leq A v_{0} \leq A u_{0} \leq v_{0}
$$

For any $x \in\left[u_{0}, v_{0}\right]$, since $A$ is a decreasing operator, we have

$$
A v_{0} \leq A x \leq A u_{0}
$$

By (2.7), we have

$$
A\left[u_{0}, v_{0}\right] \longrightarrow\left[u_{0}, v_{0}\right]
$$

It is easy to know that $\left[u_{0}, v_{0}\right]$ is a closed convex set. Since $D \subset E$ is bounded, we have $\left[u_{0}, v_{0}\right]$ is bounded. Hence, $\left[u_{0}, v_{0}\right]$ is a closed bounded convex set. Thus, Lemma $1.1 \mathrm{im}-$ plies that the operator $A$ has a fixed point in $D$.

Theorem 2.2 Let $E$ be an ordered Banach space with lattice structure, $P \subset E$ be a normal cone, and $A: E \longrightarrow E$ be a decreasing and condensing operator. Then the operator $A$ has a fixed point in $E$.

Proof For any $x \in E$, since $A: E \longrightarrow E$, we have $A x \in E$.

Since $E$ is a Banach space with lattice structure, there exists $u_{0} \in E$ such that

$$
\inf \{A x, x\}=u_{0} \text {. }
$$

That is,

$$
u_{0} \leq A x, \quad u_{0} \leq x
$$

Since $A$ is a decreasing operator, we have

$$
A^{2} x \leq A u_{0}, \quad A x \leq A u_{0}
$$

(2.8) and (2.9) show that

$$
u_{0} \leq A u_{0}
$$

Similar to the proof of (2.10), there exist $v_{0} \in E$ such that

$$
\sup \{A x, x\}=v_{0}
$$

That is,

$$
A x \leq v_{0}, \quad x \leq v_{0} .
$$

Since $A$ is a decreasing operator, we have

$$
A v_{0} \leq A^{2} x, \quad A v_{0} \leq A x .
$$


(2.11) and (2.12) show that

$$
A v_{0} \leq v_{0}
$$

(2.10) and (2.13) together with $u_{0} \leq v_{0}$ show that

$$
u_{0} \leq A v_{0} \leq A u_{0} \leq v_{0}
$$

For any $x \in\left[u_{0}, v_{0}\right]$, since $A$ is a decreasing operator, we have

$$
A v_{0} \leq A x \leq A u_{0}
$$

By (2.14), we have

$$
A\left[u_{0}, v_{0}\right] \longrightarrow\left[u_{0}, v_{0}\right]
$$

It is easy to know that $\left[u_{0}, v_{0}\right]$ is a closed convex set. Since $P$ is a normal cone of $E$, we have $\left[u_{0}, v_{0}\right]$ is bounded. Hence, $\left[u_{0}, v_{0}\right]$ is a closed bounded convex set. Thus, Lemma 1.1 implies that the operator $A$ has a fixed point in $D$.

\section{Corollaries and relative results}

Similar to the proof of Theorem 2.1, by Lemma 1.2 and Lemma 1.3, we can get the following corollaries and relative results.

Corollary 3.1 Let $E$ be an ordered Banach space with lattice structure, $D \subset E$ be bounded, and $A: D \longrightarrow D$ be a decreasing and completely continuous operator. Then the operator $A$ has a fixed point in $D$.

Corollary 3.2 Let $E$ be an ordered Banach space with lattice structure, $P \subset E$ be a normal cone, and $A: E \longrightarrow E$ be a decreasing and completely continuous operator. Then the operator $A$ has a fixed point in $E$.

Corollary 3.3 Let E be an ordered Banach space with lattice structure, $D \subset E$ be bounded, and $A: D \longrightarrow D$ be a decreasing and strict-set-contraction mapping. Then the mapping $A$ has a fixed point in $D$.

Corollary 3.4 Let $E$ be an ordered Banach space with lattice structure, $P \subset E$ be a normal cone, and $A: E \rightarrow E$ be a decreasing and strict-set-contraction mapping. Then the mapping $A$ has a fixed point in $E$.

\section{Applications}

In this section, we use Theorem 2.1 to show the existence of a solution for the uniformly elliptic differential problem. Let $\Omega$ be a bounded convex domain in $R^{n}(n \geq 2)$ whose boundary $\partial \Omega$ is assumed to be sufficiently smooth. Consider a uniformly elliptic differential operator on $\bar{\Omega}$

$$
L u=-\sum_{i, j=1}^{n} a_{i j}(x) \frac{\partial^{2} u}{\partial x_{i} x_{j}}+\sum_{i, j=1}^{n} b_{i}(x) \frac{\partial u}{\partial x_{i}}+c(x) u
$$


i.e., there exists a positive constant $\mu_{0}$ such that $\sum_{i, j=1}^{n} a_{i j}(x) \xi_{i} \xi j \geq \mu_{0}|\xi|^{2}$ for any $x \in \bar{\Omega}$ and $\xi=\left(\xi_{1}, \xi_{2}, \ldots, \xi_{n}\right) \in R^{n}$, where $a_{i j}(x)=a_{j i}(x), c(x) \geq 0$. For the sake of simplicity, we will assume that all functions $a_{j i}(x), b_{i}(x), c(x)$ are sufficiently smooth.

Considering the Dirichlet problem

$$
L u=f(x, u),\left.\quad u\right|_{\partial \Omega}=0,
$$

we have the following conclusions.

Theorem 4.1 Suppose that $f(x, u) \in C(\bar{\Omega} \times[0, \infty),[0, \infty))$, which is decreasing on $u$, then the problem (4.1) has a positive solution.

Proof It is easy to know that $E=C(\bar{\Omega})$ is a Banach space with a maximum norm $\|\cdot\|$ and it is also a lattice. Let $P=\{u \in E \mid u(t) \geq 0, \forall t \in I\}$ and $P$ be a normal cone in $E$. It is well known (see $[1,10]$ ) that the solution of the Dirichlet problem (4.1) is equivalent to the fixed point of the integral operator $A$

$$
A u(x)=\int_{\bar{\Omega}} G(x, y) f(y, u(y)) d y,
$$

where $G(x, y)$ denotes the Green function of a differential operator L with boundary condition $\left.u\right|_{\partial \Omega}=0$. It is also well known that $G(x, y)$ satisfies the following inequality:

$$
0<G(x, y)<\left\{\begin{array}{ll}
K_{0}|x-y|^{2-n}, & n>2, \\
K_{0}|\ln | x-y||, & n=2
\end{array} \quad(x, y \in \Omega, x \neq y) .\right.
$$

Hence, the linear integral operator

$$
B v(x)=\int_{\bar{\Omega}} G(x, y) v(y) d y
$$

is a completely continuous operator from $E$ into $E$. Clearly, the superposition operator $F \phi(x)=f(x, \phi(x))$ that maps $P$ into $P$ is continuous and bounded. Therefore, the operator $A=B F$ that maps $P$ into $P$ is completely continuous, and thus $A$ is condensing.

Moreover, the mapping $A$ is decreasing in $u$. In fact, by hypotheses, for $u \geq v$,

$$
f(t, u(x)) \leq f(t, v(x))
$$

implies that

$$
\begin{aligned}
(A u)(x) & =\int_{\bar{\Omega}} G(x, y) f(y, u(y)) d y \\
& \leq \int_{\bar{\Omega}} G(x, y) f(y, v(y)) d y=(A v)(x), \quad x \in \bar{\Omega},
\end{aligned}
$$

so $A$ is decreasing.

So, the condition of Theorem 2.1 holds, Theorem 4.1 is proved. 
Competing interests

The authors declare that they have no competing interests.

\section{Authors' contributions}

$\mathrm{XL}$ carried out the the main theorem and the main conclusion. ZW carried out the application of the main theorem. All authors read and approved the final manuscript.

\section{Acknowledgements}

The first author was supported financially by the NSFC (71240007, 11001151), NSFSP (ZR2010AM005).

Received: 2 December 2012 Accepted: 14 January 2013 Published: 30 January 2013

\section{References}

1. Guo, D: Positive fixed points and eigenvectors of noncompact decreasing operators with applications to nonlinear integral equations. Chin. Ann. Math., Ser. B 4, 419-426 (1993)

2. Nieto, JJ, Rodríguez-López, R: Existence and uniqueness of fixed point in partially ordered sets and applications to ordinary differential equations. Acta Math. Sin. 23(12), 2203-2212 (2007)

3. O'Regan, D, Petrusel, A: Fixed point theorems for generalized contractions in ordered metric spaces. J. Math. Anal. Appl. 341, 1241-1252 (2008)

4. Nieto, JJ, Pouso, RL, Rodríguez-López, R: Fixed point theorems in ordered abstract spaces. Proc. Am. Math. Soc. 135, 2505-2517 (2007)

5. Sadarangani, K, Caballero, J, Harjani, J: Contractive-like mapping principles in ordered metric spaces and application to ordinary differential equations. Fixed Point Theory Appl. 2010, Article ID 916064 (2010)

6. Nieto, JJ: An abstract monotone iterative technique. Nonlinear Anal. 28, 1923-1933 (1997)

7. Wu, Y: New fixed point theorems and applications of mixed monotone operator. J. Math. Anal. Appl. 341, 883-893 (2008)

8. Gnana Bhaskar, T, Lakshmikantham, V: Fixed point theorems in partially ordered metric spaces and applications. Nonlinear Anal. TMA 65(7), 1379-1393 (2006)

9. Sadovskii, BN: A fixed point principle. Funct. Anal. Appl. 1, 151-153 (1967)

10. Gnana Bhaskar, T, Bose, RK: Some Topics in Nonlinear Functional Analysis. Wiley, New Delhi (1985)

11. Darbo, G: Punti uniti in trasformazioni a condominio non compatto. Rend. Semin. Mat. Univ. Padova 24, 84-92 (1955)

doi:10.1186/1687-1812-2013-18

Cite this article as: Li and Wang: Fixed point theorems for decreasing operators in ordered Banach spaces with

lattice structure and their applications. Fixed Point Theory and Applications 2013 2013:18

\section{Submit your manuscript to a SpringerOpen ${ }^{\circ}$ journal and benefit from:}

- Convenient online submission

- Rigorous peer review

Immediate publication on acceptance

Open access: articles freely available online

- High visibility within the field

- Retaining the copyright to your article 